\title{
Obturator Neuropathy Combined with Guillain-Barre Syndrome
}

\author{
Çağlar Macit ${ }^{*}$, Neda Taner ${ }^{2}$, Ozan Emre Eyüpoğlu ${ }^{3}$ \\ 1 Istanbul Medipol University, School of Pharmacy, Department of Pharmacology, Istanbul, Turkey \\ 2 Istanbul Medipol University, School of Pharmacy, Department of Clinical Pharmacy, Istanbul, Turkey \\ 3 Istanbul Medipol University, School of Pharmacy, Department of Biochemistry, Istanbul, Turkey
}

\begin{abstract}
Obturator neuropathy caused by entrapment of obturator nerve is a disease with numbness, pain and weakness in legs. Guillain-Barre syndrome is an immune-mediated peripheral polyneuropathy characterized by nearly same symptoms.

51-year-old man presented with severe shortness of breath. He was admitted to ICU and treated with mechanical ventilation followed complete blood count (CBC). We observed inflammation, started required medications and, we provided improvement in a short time. When patient was visited, he explained numbness, pain in hip area, muscle weakness in all extremities. Cervical MRI and nerve conduction tests were performed. MRI showed oedema in sciatic nerve and entrapment of obturator canal, resulted to obturator neuropathy and nerve conduction tests revealed GBS. He was treated with plasmapheresis and IVIG, he was discharged without neurological recovery. The patient consent was taken orally.

In conclusion, treatments decreased complaints and provided to walk short distances. Reinnervation signals were observed in nerve conduction test.
\end{abstract}

Keywords: Guillain-Barre Syndrome, Obturator Neuropathy, IVIG, Nerve Conduction Test

\section{INTRODUCTION}

Obturator neuropathy $(\mathrm{ON})$ is an illness presenting with sensory loss, pain or weakness with leg adduction. Guillain-Barre Syndrome (GBS) has similar symptoms. The most determinative diagnostic parameters are nerve conduction in both diseases. ${ }^{1,2}$ Therefore, GBS may also leads to ON whether it was diagnosed late. There have been lots of studies in literatures about these illnesses,

*Corresponding Author: Çağlar Macit, e-mail: cmacit@medipol.edu.tr Çağlar Macit ORCID Number: 0000-0002-5532-2395

Neda Taner ORCID Number: 0000-0002-6141-8676

Ozan Emre Eyupoğlu ORCID Number: 0000-0002-4449-0537

(Received 23 May 2019, accepted 23 September 2019) 
separately. However, this is the first report where GBS and ON are seen in combination. Our objective is to show IVIG therapy is effective for the treatment of both GBS and ON diseases. This report mentions about on GBS combined with Obturator neuropathy. This combination is the first in the globe according to the literature. Additionally, there is an important data that IVIG therapy which is used to treat GBS disease, also improved Obturator neuropathy.

\section{METHODOLOGY}

A 51-year-old man was admitted with loss of consciousness and shortness of breath (SOB). On hospitalization, his family also explained about muscle weakness and pain while sitting and walking. After admission to intensive care unit (ICU) of hospital, intubation, fluid replacement therapy and mechanical ventilation were provided. His first complete CBC showed increased CRP $(39,5 \mathrm{mg} / \mathrm{L})$ with leukocytosis $\left(15,2^{*} 10^{9} / \mathrm{L}\right)$ and thrombocytosis $\left(663^{*} 10^{3}\right)$. All these complaints made us think about an inflammatory disease. First, IV isotonic $\mathrm{NaCl}$ $50 \mathrm{~mL}$ for hydration, teicoplanin $400 \mathrm{mg}$ IV 3x1 for possible sepsis, salbutamol nebulizer for SOB, paracetamol IV for pain, pregabalin NG for neuropathic pain and NG nutrition because of his unconsciousness were administered.

A few hours later patient was recovered. Next day, he explained loss of feel, $\mathrm{SOB}$, weakness in his extremities and severe pain in his hip. In that condition, we decided to perform full motor and sensorial studies and cervical MRI. Clinical and electrodiagnostic findings showed GBS with axonal damage at ligaments that seen in Table 1. 
Table 1. Clinical and electrodiagnostic findings

Motor nerve studies

\begin{tabular}{|c|c|c|c|c|c|c|}
\hline Nerves and Record sites & \multicolumn{2}{|c|}{$\begin{array}{c}\text { Latency } \\
(\mathbf{m s})\end{array}$} & \multicolumn{2}{c|}{ Amplitude (mV) } & \multicolumn{2}{c|}{$\begin{array}{c}\text { NCV } \\
\text { (m/sec) }\end{array}$} \\
\hline Median Nerve & Left & Right & Left & Right & Left & Right \\
\hline Wrist & 4,1 & 3,8 & 6,1 & 3,2 & - & - \\
\hline Elbow & 8,5 & 8,5 & 3,8 & 2,0 & 52,1 & 49,3 \\
\hline Ulnar Nerve & & & & & & \\
\hline Wrist & & 4,3 & & 2,4 & & - \\
\hline Elbow & & 9,3 & & 1,9 & & 66,3 \\
\hline Peroneal Nerve & & & & & & \\
\hline Ankle & & 4,0 & & 3,2 & & - \\
\hline Fibula head & & 9,6 & & 3,1 & & 50,1 \\
\hline Pop.fos. & & 11,9 & & 2,8 & & 42,9 \\
\hline Tibial Nerve & & & & & & \\
\hline Ankle & & 4,3 & & 7,4 & & - \\
\hline Pop.fos. & & 12,2 & & 6,5 & & 46,7 \\
\hline
\end{tabular}

Sensory nerve studies

\begin{tabular}{|c|c|c|c|c|c|c|c|c|}
\hline Nerve & $\begin{array}{c}\text { Recod } \\
\text { site }\end{array}$ & $\begin{array}{c}\text { Stimulation } \\
\text { Site }\end{array}$ & \multicolumn{2}{|c|}{$\begin{array}{c}\text { Latency } \\
(\mathbf{m s})\end{array}$} & \multicolumn{2}{|c|}{ Amplitude $(\boldsymbol{\mu V})$} & \multicolumn{2}{|c|}{$\begin{array}{c}\text { NCV } \\
(\mathbf{m} / \mathbf{s e c})\end{array}$} \\
\hline $\begin{array}{c}\text { Sural } \\
\text { Nerve }\end{array}$ & Wrist & Mid-calf & Left & Right & Left & Right & Left & Right \\
\hline & & & 2,9 & & 10,7 & & 48,3 \\
\hline $\begin{array}{c}\text { Median/ } \\
\text { Ulnar/ } \\
\text { Radius }\end{array}$ & Wrist & Index finger & & 2,7 & & 12,0 & & 44,4 \\
\hline & & $5^{\text {th }}$ digit & & 2,3 & & 8,3 & & 45,7 \\
\hline
\end{tabular}

\section{F-wave Studies}

\begin{tabular}{|c|c|c|c|c|c|}
\hline \multirow{2}{*}{ Nerve } & \multirow{2}{*}{ Record site } & \multirow{2}{*}{$\begin{array}{c}\text { Stimulation } \\
\text { site }\end{array}$} & & \multicolumn{3}{|c|}{ Latency (ms) } \\
\cline { 4 - 6 } & & M wave & F wave & F-M wave \\
\hline Right Median & APB & Wrist & 4,08 & 30,83 & 26,75 \\
\hline Right UInar & ADM & Wrist & 3,25 & 35,17 & 31,92 \\
\hline Right Tibial & AH & Ankle & 3,83 & 51,00 & 47,17 \\
\hline
\end{tabular}

ADM: Abductor digiti minimi; AH: Abductor hallucis; APB: Abductor pollicis brevis; NCV: Nerve conduction velocity; Pop.fos.: Popliteal fossa 
Cervical MRI expressed common T2 signal increase in all muscles and fascial areas, but mainly at the obturator externa and interna. Furthermore, oedema was also observed in sciatic nerve (Figure 1).

a)

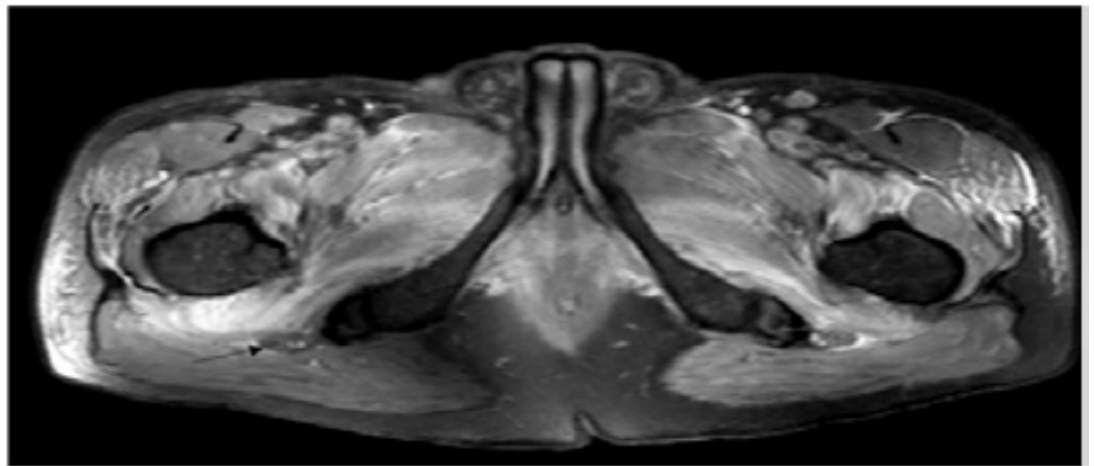

b)

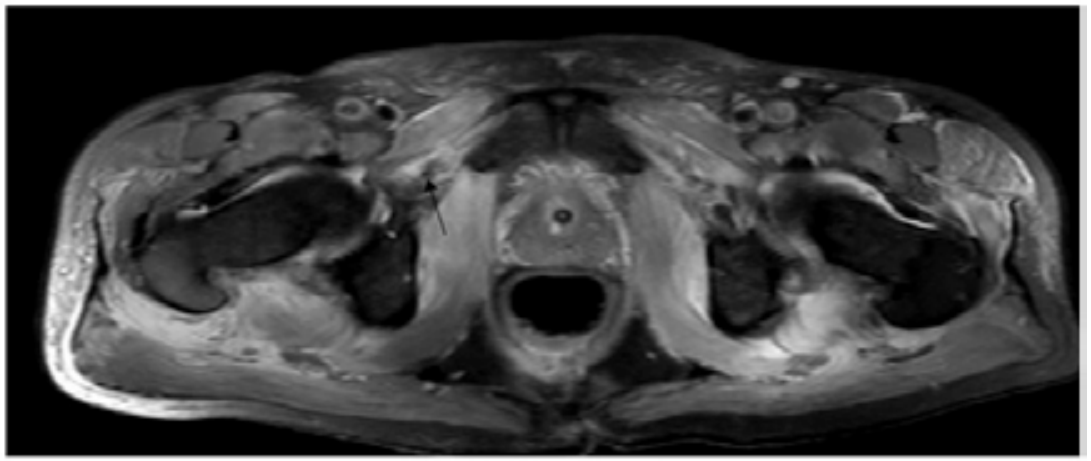

Figure 1. a) Entrapment of obturator channel; b) Oedema in sciatic nerve

We thought that these situations occurred as a secondary change to polyneuropathy associated with GBS. Immediately, plasmapheresis treatment was administered for 7 days. Plasmapheresis therapy resulted in clinical improvement and patient was discharged from hospital. However, due to insufficient neurological healing, he admitted rehabilitation programs. 3 weeks later, he came back with same complaints and then given $5 \mathrm{~g}$ x $1 \times 5$ vials/daily dose of IV immunoglobulin (IVIG) therapy. Motor and sensorial studies were repeated, and it was observed that motor abilities and other symptoms of patient became better. However, little progression was determined in axonal damage. After from 5-day-follow up process, he was discharged to home with his medications He used pregabalin for peripheral neuropathy, salbutamol inhaler for SOB and prednisolone for inflammation of obturator externa and interna. 5 months later he came back to neurology ward and he was controlled to see the progression. He was observed polyneuropathy with subacute axonal damage. 
However, it was significantly better than before. Moreover, reinnervation potentials were also observed in the examination mainly in obturator nerves. Finally, oedema in sciatic nerve and inflammation of obturator joint was treated completely and he was able to walk short distances using 2 crutches.

\section{RESULTS AND DISCUSSION}

Obturator neuropathy (ON) is a severe and common illness. There are plenty of conditions that cause also lead to suppression of nerve and results in obstetrical ON.3.4 Entrapment of nerve should be considered as cause of pain, loss of sense and weakness in muscle. Guillain-Barre syndrome (GBS) is a progressive and immune-mediated peripheral polyneuropathy characterized by motor and sensory weakness. Documentations reports estimated incidence of GBS for all ages combined vary from 0.16 to 3.0 per 100 ,ooo person-years. ${ }^{5}$ As we all know basic complaints of GBS is managed by use of IV immunoglobin therapy and plasmapheresis. Detailed anamnesis, physical examination and clinical findings are very important to recognise the illnesses correctly as soon as possible, because, the prognosis of both GBS and ON is dependent on early diagnosis. ${ }^{6}$ Motor and sensory tests and MRI scanning should be done. Additionally, if it is possible, diagnosis should be supported by magnetic resonance neuroimaging (MRN).7 In studies performed by Riva et al. and Van Asseldonk et al., IV immunoglobulin treatment healed motor axonal neuropathy transiently and marginally. ${ }^{8,9}$ We also observed the similar clinical ameliorations in our patient. Obturator neuropathy has similar symptoms; flaccid weakness of lower extremities, loss of sense and fulminant pain while sitting. To improve these, analgesics, pregabalin (for peripheral neuropathy) and physiotherapy are suggested..$^{10}$ Addition to these, it should be studied in future whether IV immunoglobulin may be beneficial in both GBS and ON treatment.

In conclusion, not only symptoms and diagnosis process, but also treatment of both diseases is very similar. In our report, patient has two separate polyneuropathic diseases. However, we diagnosed them early and began to treat. Although obturator joint inflammation and sciatic nerve oedema were treated successfully, obturator neuropathy was not recovered completely. Although this condition, patient could walk short distances.

On the other hand, unfortunately, GBS was not treated completely. However, reinnervation signals were observed at electrophysiological test.

This report is very interesting and important. Since according to our knowledge, obturator neuropathy combined GBS case was encountered for the first time and we showed that IVIG also heals obturator neuropathy beside GBS. 


\section{CONFLICT OF INTEREST}

The authors have no potential conflict of interest relevant to this article.

Informed Consent: Verbal informed consent was obtained from patient and his parents who participated in this study.

\section{ACKNOWLEDGEMENTS}

We declared that this study has received no financial support. We had required permissions for this study. We also thank to Neurology specialist Dr. Zeki Kilic (Nigde Omer Halisdemir University Training and Research Hospital, Turkey) for his supervision in this study.

\section{ETHICAL PUBLICATION STATEMENT}

We confirm that we have read and understood the Journal's position on issues involved in ethical publication and affirm that this report is consistent with those guidelines. This study has ethical approval with the protocol number of 594/01.08.2019. 


\section{REFERENCES}

1. Akıl, E.; Varol, S.; Taşkın, A.; Arıkanoğlu, A.; Tamam, Y.; Öztürk, Ü. Guillain-Barre sendromunda klinik ve demografik özellikler. Dicle Univ. Tip. Fakul. Derg. 2015, 41, 707-711.

2. Korinthenberg, R.; Schessl, J.; Kirshner, J. Clinical presentation and course of childhood Guillain-Barre Syndrome: a prospective multicentre study. Neuropediatrics 2007, 30, 10-17.

3. Bradshaw, C.; McCrory, P.; Bell, S.; Brukner, P. Obturator neuropathy: a cause of chronic groin pain in athletes. Am. J. Sports Med. 1997, 25, 402-408.

4. Craig, W. S.; Clark, J. M. Obturator palsy in the newly born. Arch. Dis. Child. 1962, 37, 661-662.

5. McGrogan, A.; Madle, G.C.; Seaman, H.E.; de Vries, C.S. The epidemiology of GuillainBarré syndrome worldwide. A systematic literature review. Neuroepidemiology 2009, 32, $150-163$.

6. Jin, H. Y.; Lee, K. A.; Kim, S. Y.; Park, J. H.; Baek, H. S.; Park, T. S. A Case of Diabetic Neuropathy Combined with Guillain-Barre Syndrome. Korean J. Intern. Med. 2010, 25, 217-220.

7. Muniz, Neto, F.J.; Kihara, Filho, E.N.; Miranda, F.C.; Rosemberg, L.A.; Santos, D.C.B.; Taneja, A.K. Demystifying MR Neurography of the Lumbosacral Plexus: From Protocols to Pathologies. BioMed Res. Int. 2018, 2018, 9608947.

8. Riva, N.; Gallia, F.; Iannaccone, S.; Corbo, M.; Terenghi, F.; Lazzerini, A. Chronic motor axonal neuropathy. J. Peripher. Nerv. Syst. 2o11, 16, 341-346.

9. Van Asseldonk, J. T.; Franssen, H.; Van den Berg-Vos, R.M.; Wokke, J.H.; Van den Berg, L.H. Multifocal motor neuropathy. Lancet Neurol. 2005, 4, 309-319.

10. Tipton, J. S. Obturator neuropathy. Curr. Rev. Musculoskelet. Med. 2oo8, 1, 234-237. 